\title{
Electroanalysis from the past to the twenty-first century: challenges and perspectives
}

\author{
José Manuel Díaz-Cruz ${ }^{1,2} \cdot$ Núria Serrano ${ }^{1,2} \cdot$ Clara Pérez-Ràfols $^{1,3} \cdot$ Cristina Ariño $^{1,2} \cdot$ Miquel Esteban $^{1,2}$ (D)
}

Received: 3 June 2020 / Revised: 15 June 2020 / Accepted: 16 June 2020 / Published online: 21 June 2020

(C) Springer-Verlag GmbH Germany, part of Springer Nature 2020

\begin{abstract}
A personal mini-review is presented on the history of electroanalysis and on their present achievements and future challenges. The manuscript is written from the subjective view of two generations of electroanalytical chemists that have witnessed for many years the evolution of this discipline.
\end{abstract}

Keywords Electroanalysis $\cdot$ Electrochemistry $\cdot$ History of electroanalysis

\section{Introduction: a particular view}

Our research group at the University of Barcelona was founded by the late Prof. Enric Casassas and has been active in the field of electroanalysis since the 1980s. Some of us (the older ones) remember how we have witnessed the success and decadence of mercury electrodes and have entered an uncertain but exciting period when many electrode materials, supports and modifications compete with each other to lead the emerging world of electrochemical (bio)sensors. Fortunately, the younger members of our team are fully involved in the new trends and maybe they will see a new golden age of electroanalysis. This huge transformation of the electrodes has been accompanied by a drastic evolution of the data treatment, starting from classical electrochemical hard modelling, progressively incorporating chemometric strategies and nowadays running towards data fusion and big data. Thus, the commemoration of the 65th anniversary of Prof. Fritz Scholz, a great researcher and divulgator of electrochemistry,

Miquel Esteban

miquelestebanc@ub.edu

1 Department of Chemical Engineering and Analytical Chemistry, University of Barcelona, Martí i Franquès 1-11, E08028 Barcelona, Spain

2 Water Research Institute (IdRA) of the University of Barcelona, Barcelona, Spain

3 Department of Chemistry, School of Engineering Science in Chemistry, Biochemistry and Health, KTH Royal Institute of Technology, Teknikringen 30, SE-10044 Stockholm, Sweden is a good time for us to explain our modest view about the recent progress and the perspectives of electroanalysis. For this purpose, we will mix both the experience and the enthusiasm of several generations of researchers of our group sharing a common fascination for the movements of electrons and ions inside electrochemical circuits.

\section{Prehistory and history: the age of metals}

Many chemists and historians consider Alchemy as the antecessor of chemistry. Alchemists searched for the philosopher's stone and, in their way, they found things far more exciting than a stone able to cause a fast devaluation of the gold's price. It was Boyle, and Lavoisier and Dalton, and many others who invented the writing in chemistry (with signs, measurements and laws) and moved this discipline from prehistory to history [1].

In our particular mythology, electrochemists can invoke the Greek name 'elektron', given to amber and related to electricity since ancient times due to the electrostatic properties of such material. Indeed, this tradition inspired George Johnstone Stoney in 1894 to give the name 'electron' to the negative subatomic particle that had been predicted by many researchers up to date and was effectively discovered 3 years later, in 1897, by Joseph John Thomson. From the Greek people rubbing amber to the first electron caught by J.J. Thomson, there are many centuries of rude electrochemical prehistory, which we may convey that end with the pioneering frog's legs by Galvani. Then, electrochemical writing was established by a large deal of scientists like Volta, Ampère, Ohm or Faraday who not only invented a new language but 
also gave their names to the units of the brand-new electrochemical magnitudes [2-8]. And, since that time, electrochemistry has been essential for the progress of science and the welfare of humanity [9].

The history of electroanalysis started not much later than that of electrochemistry, just when analytical chemists discovered that the new quantitative laws such as these by Nernst or Faraday could be effectively used to provide information about the chemical composition of samples [8]. From these early times, two separated branches arose: on the one hand, potentiometry focused on thermodynamics and potentials and, on the other hand, amperometry and voltammetry focused on kinetics and currents [10]. It is the classical antagonism between the static view of the universe by Parmenides ("change is impossible") and the dynamic view by Heraclitus ("everything flows"). If they were electrochemists today, Parmenides would surely study potentiometry and Heraclitus, voltammetry.

\section{The age of mercury (and membranes)}

In human prehistory and history, the technological progress of civilizations is often measured in terms of the materials employed. Thus, for instance, it is famous the historical evolution of metals used for weapons in the sequence copper, bronze, iron and steel. In the early times of electroanalysis, many metals were tested as suitable materials for electrodes (our weapons) and, surprisingly, the best candidates were not those historically used for swords, but those preferred for jewellery: silver, gold and platinum. Also, a less noble material was added to the list: carbon in different physical states. Even today, these materials constitute the basis of conventional solid electrodes. Their main drawback, however, is their trend to get dirty and to lose their electrochemical properties as both reagents and products adsorb onto the surface. To avoid this, tedious and time-consuming cleaning procedures are required including extensive polishing and electrochemical cycling in the presence of redox agents [11].

As time went by, new materials were found that clearly improved the performance of such electrodes. On the potentiometric side, the glass membrane sensitive to $\mathrm{H}^{+}$-ions founded a dynasty of ion-selective electrodes based on a great diversity of membranes and charge carriers. On the voltammetric side, the invention of polarography by Jaroslav Heyrovský in 1922 raised an unexpected metal such as mercury to the top of electrode materials [12]. Although mercury was already known by ancient civilizations (and even associated to a planet), it had been just a liquid curiosity for alchemists and eccentric emperors until scientists started to use it in barometers, thermometers and polarographs.

For many years, membranes have been ruling potentiometric measurements, and they have been miniaturized and combined to become potentiometric sensors and even potentiometric sensor arrays. They have the advantage of an easy acquisition and treatment of data (based on Nernst equation) but the drawback of a low sensitivity [13-15].

In contrast, polarography offered much lower detection limits for trace analysis and, moreover, a greatly extended cathodic working range (ideal for metal ions) and a constantly renewed surface with every new drop that made unnecessary the typical cleaning procedures of solid electrodes [16-18]. As a drawback, the data treatment is quite more complex than in potentiometry, but this was not a problem for several generations of electrochemists who developed a large set of equations for every particular case by smart combination of laws for electrochemical equilibrium, kinetics and mass transport. This is what some researchers call electrochemical hard modelling $[2,19]$.

\section{The twilight of mercury and the raise of screen- printed electrodes}

For a long time, mercury electrodes and hard modelling were a key reference in electroanalysis. It is true that membrane electrodes were quite successful at determining relatively high concentrations of analytes, and that solid electrodes were the ideal platform to develop promising modifications and to carry out amperometric detection in flow systems. But mercury electrodes were the most reproducible and the best choice for determining heavy metal ions by means of stripping techniques [20].

But in the beginning of the twenty-first century, the increasing concern about the toxicity of mercury promoted restrictive regulations that progressively discouraged researchers from using it. Fortunately, by that time, the alternatives to mercury were far more exciting than some decades before [21]. Ultramicroelectrodes [22], rotating disk electrodes [23], composite solid electrodes [24] and new and promising solid materials like boron-doped diamond (BDD) [25] were especially versatile and offered far more possibilities of modification than some years before. Many relevant characteristics of mercury such us the wide cathodic range and the ability to accumulate reduced metal ions could be acceptably reproduced with bismuth and antimony films coated on solid electrodes (typically made of glassy carbon) [26-28]. And finally, commercial screen-printed electrodes (SPE) evolved as a costless and versatile alternative to conventional solid electrodes. These devices are the result of printing the components of an electrochemical circuit (namely the working, reference and auxiliary electrodes and the corresponding connectors) into a plastic or ceramic support by using inks containing the materials of such components (silver, gold, graphite...). A further curing of the inks at high temperature and the coverage of sensible circuits with an insulator layer finish a device containing all three electrodes together, so that they can measure either in a single drop, immersed into a 
solution or attached to a flow system. Although the surface of the working electrode of a SPE unit is not as perfect as that of a mirror-like polished solid electrode, it has the advantage of being disposable and cheap, so that after a series of measurements, the device can be replaced by a brand-new unit with similar performance $[29,30]$. Obviously, this avoids the recurrent polishing, cleaning and activation protocols of conventional solid electrodes, and makes SPE very attractive for using them either in its original form or as a support for a great diversity of modifications. Then, when it was clear the wreck of mercury, many polarographists like us tried to survive by firmly grabbing screen-printed devices and most of us succeeded [30-35].

\section{The importance of being a sensor}

While most electrochemists were playing with membranes, mercury drops, solid electrodes and cumbersome equations in Laplace's domain, a huge technological progress was made in the fields of spectroscopy and chromatography. Key milestones in this progress were the development of inductively coupled plasma (ICP) techniques and the coupling of mass spectrometry (MS) to gas (GC) and liquid (LC) chromatography. We should not forget, either, the advances in X-ray fluorescence (XRF) and near-infrared spectroscopy (NIR). Although, in general, such techniques were not especially emphasized during the academic degrees in chemistry, they have become really smart tools for the analysis of solid samples. Then, the obsolescence of mercury took place at the same time that extremely powerful techniques like ICP-OES, ICP-MS, $\mathrm{XRF}, \mathrm{GC}-\mathrm{MS}$ or LC-MS claimed to be able to solve any analytical problem related to atoms (ICP-OES, ICP-MS, $\mathrm{XRF}$ ) or molecules (GC, LC-MS) [36-38]. Apparently, there was no place for us, the old and poor electroanalytical chemists.

This could have been a perfect storm for electroanalysis or, better, like the meteorite that extinguished dinosaurs and menaced with the end of life in the Earth. But life survived then becoming small and fast and, hence, little mammals conquered the lost kingdom of big reptiles. Thus, in a similar way, electroanalytical devices survived trying to be small, portable, cheap and fast, i.e. becoming electrochemical sensors.

Of course, by that time, electrochemical sensors had been existing for several decades and many research groups had made valuable contributions to the field $[39,40]$, the jewel of the crown being the glucose sensor for diabetics [41]. But we believe that by that time, a significant number of electroanalytical chemists (including us) started to focus on sensors as the only opportunity to remain competitive in analytical chemistry. And, for sure, it was a good moment to move to this topic. Transduction had always been the biggest advantage of electrochemical sensors, since they directly produce signals in electric form, which is the goal of all sensors independently of their operating principle. But now, electrochemical (bio)sensors have additional advantages related to the reception, since a large deal of biomolecules and nanomaterials has been incorporated to electrochemical research in the last years [42]. Moreover, SPE and other mass production supports are strong allies in the task of making sensors simple, smart and cheap enough to be effective in the screening and in situ monitoring of samples.

\section{Electrochemical data treatment: from big equations to big data}

The analysis of electrochemical data has also experienced sensitive changes in the last years. The data analysis of potentiometric measurements (based on Nernst's equation) and the amperometric detection in LC had always been relatively easy. Hence, analytical electrochemists working in these subjects were more concerned about experimental developments and problems than about data treatment.

In contrast, researchers working in more sophisticated techniques like voltammetry or chronopotentiometry had to deal with many physico-chemical processes simultaneously taking place during the measurements. The most ambitious strategy for that is hard modelling [2]. This consists on elaborating a model with rigorous equations describing the electrochemical kinetics and equilibria at the electrode surface, the homogeneous kinetics and equilibria inside the bulk solution, the mass transport towards the electrode and even undesired phenomena such as the adsorption of species onto the electrode surface. This usually results in a system of partial derivative equations as a function of position and time, which, in the simplest situations, can be analytically solved by means of Laplace's transformation to yield classical milestones of electrochemistry such as the Cottrell's equation. Nevertheless, as the complexity of the considered system increased, the set of derivative equations became more complicated and, often, impossible to solve analytically. Then, two different approaches arose. On the one hand, researchers mostly from the physical chemistry branch used numerical methods to solve the equations and simulate the expected evolution of signals as functions of different experimental variables. In some cases, they were even able to extract relevant parameters from the numerical fitting to experimental signals. On the other hand, researchers mostly from analytical chemistry used approximate classical equations, extracting from them a few overall parameters like limiting and peak currents or half-wave and peak potentials to explain the evolution of experimental signals. The method by DeFord and Hume to evaluate stability constants of metal complexes is a good example of this approach [43]. The most empirical analysts, however, just forgot any electrochemical background and simply used the signals (mostly the peakshaped ones) as far as their height or area was proportional 
to the concentration of analyte. For this purpose, the standard addition method was used as a calibration strategy quite insensitive to matrix effects and secondary electrochemical phenomena.

But when the topics addressed by electroanalysis got deeper into fields such as bioanalysis, environment or food science, both strategies went to a crisis, overwhelmed by the large complexity of experimental systems and the large deal of undesired phenomena impossible to model by using the classical hard modelling approach. Even the most empirical strategies suffered when they had to face the presence of many overlapping signals and cumbersome baselines. For many years, electrochemistry had been working with large and intricate equations ruled by a few relevant physicochemical parameters (e.g. diffusion coefficients, stability constants and concentrations) and applied to a restricted set of experimental values (e.g. peak height, position and width) to guess the values of such parameters. Fortunately, in the 1990s, a new strategy, based on chemometrics, entered the domain of electroanalytical data $[44,45]$. This is known as soft modelling and essentially attempts to take advantage not only from relevant parameters but also from the whole experimental signal. Then, it uses simple, matrix-based models to solve analytical problems (discrimination, calibration) with as little information as possible about the electrochemistry involved. In this way, 'classical' chemometric techniques like principal component analysis (PCA) or partial least squares (PLS) started to be popular in electroanalysis for data exploration, sample discrimination and multivariate calibration [46]. A less drastic approach was proposed with the use of multivariate curve resolution by alternating least squares (MCR-ALS), which allows one to impose a series of constraints during the iterative optimization to keep the electrochemical meaning of the concentrations and the pure signals computed [47].

Therefore, it is clear that the progressive replacement of hard by soft modelling is irreversible and definitely we are going from big equations to big data. In addition, it should be taken into account that electroanalytical data will be every time bigger, because electrochemical sensors have started to operate in groups. This is the case of potentiometric electronic tongues, which use a set of membrane electrodes not especially selective to any substance in particular but with cross response to relevant analytes, so that the combination of their responses can allow the discrimination of samples or the quantification of substances $[15,48]$. Voltammetric electronic tongues operate in the same way, but they work with a much bigger number of values (a full voltammogram with hundreds of points instead of a single potential) [49]. Hybrid tongues and data fusion combining different types of measurements still involve more data, and if in the future there is an extensive deployment of wireless sensor networks, they will generate for sure a volume of information that would deserve the designation of big data.
But the challenges of data treatment are not only related to the high volume of information but also to its complexity. Electrochemical data use to be non-linear and techniques like PCA, PLS or MCR-ALS are conceived for linear data. Therefore, advanced approaches like artificial neural networks (ANN) or supporting vector machines (SVD) will be required. Moreover, important problems will have to be addressed such as matrix effects and signal drifts to increase the reliability of sensor measurements [49].

\section{An electrochemical crossroad}

Nowadays, electroanalytical chemists work to ensure that sensors could be a suitable alternative to the extremely powerful ICP-MS and GC,LC-MS. techniques. Obviously, sensors cannot compete with them in terms of accuracy, selectivity, multianalyte features or detection limits but, as already mentioned, sensors have the advantage of being small, fast, cheap and portable, which means ideal for screening and on-site monitoring. We have believed that for a long time, but we are still more convinced now that we are confined at home because of covid-19. In this critical scenario, we see that authorities are not looking for expensive instrumentation placed in centralized laboratories. On the contrary, they try to get as much as possible test devices enough simple and fast to detect infected people in all possible places (in hospitals, supermarkets, companies, particular houses...). This shows that, in many situations, it is much more convenient to bring the analysis to the sample than to bring the sample to the analysis, even if it is made by the most sophisticated instruments. In this sense, biosensor devices could provide improvements in the weak points offered by the reference polymerase chain reaction (PCR) technique for genomic analysis contributing to the diagnosis of diseases and health emergencies like the current one [50]. For all the above-mentioned, it is not surprising that, in the last years, journals devoted to sensors have clearly surpassed prestigious journals of general analytical chemistry, spectroscopy and chromatography in the impact factor ranking.

And we talk about an electrochemical crossroad because electrochemical sensors can progress in many directions and with many strategies. Probably one of the most profitable from both scientific and economical points of view is that, previously introduced, of biosensors [51], which take advantage of the intimate relationship between electrons and biology [52] and between ions and biomembranes [53], and is especially focused on biomedical applications [54]. Especially productive is the attempt to develop the electroanalytical version of commercial immunoassays like the widespread enzymelinked immunosorbent assay (ELISA) or even to play with DNA fragments to compete with the more sophisticated biochemical methodology of PCR as it was previously postulated by the covid-19 diagnosis. For this purpose, many recent tools 
are available to researchers such as SPE, nanoparticles, nanomaterials, magnetic beads, micromachines and a large deal of strategies to immobilize biomolecules onto the electrode surface [55-57]. In comparison with traditional biomedical methodologies, these approaches generally decrease the analysis time, but present similar drawbacks related to the fragility of biological materials that have to be preserved in the refrigerator for a limited time before their date of expiry. As for the cost of the new methods, they should be similar to these of existing methodologies (biological materials are the most expensive part in both cases), but at the moment, it is difficult to say because, despite the large investment in research on this topic not many biosensor developments have been able to reach the market. A costless and more robust option has been investigated for some time in the form of electrodes modified with molecularly imprinted polymers (MIP) [58], which generate sensitive cavities in a polymeric matrix by insertion of template molecules of similar structure as that of the analytes. If MIP were able to demonstrate similar performance as natural antibodies, they would be for sure the definitive choice, as they are much cheaper and durable and do not need special conditions of preservation, but we suspect that we will have to wait a long time to see that.

But not all electroanalytical research is made in the field of bioanalysis. Environmental samples are also quite demanding, especially since the increasing concern about persistent and emerging contaminants. Moreover, climate change has emphasized the need of reusing wastewaters and this requires a rigorous, fast and on-site analysis of the reclaimed water in wastewater treatment plants. Among key pollutants, not only usual analytes like heavy metal ions or pesticides are considered but also emerging substances such as UV filters, pharmaceutical products or anticorrosive agents. For these contaminants, voltammetry at screen-printed electrodes is yielding promising results, especially when preconcentration at the electrodes is possible, which allows quite low detection limits by means of stripping techniques $[59,60]$. In some cases, preconcentration can be made by redox processes or adsorption at the bare electrode, but this usually requires some kind of selective materials that can be bismuth or antimony films, metal chelators, peptides, and nanoparticles. In this research area, carbon-based nanomaterials like graphene, carbon nanotubes or carbon nanofibers have been very useful, either by themselves or as supports for further modifications [61]. Moreover, conducting polymers [62], titanate 2D-nanosheets [63], structured nanoallotropic forms of other elements different from carbon such as of the pnictogen group [64], or especially inert materials like boron-doped diamond (BDD) [25] may play a significant role in the future. Although not so much recognized and funded as biosensor strategies, these inorganic approaches have the advantage of a low price and a high durability, which makes them especially suitable for on-site monitoring in natural waters or in wastewater treatment plants.
Process control in industrial scenarios could also take advantage of these strategies for on-line monitoring of critical steps inside the production chain.

Another interesting field where electroanalysis can be useful is food science. Potentiometric sensors first and voltammetric devices later showed that the assembly of poorly selective sensors in the form of electronic tongues can be very effective in the discrimination of food samples of different protected designations of origin (PDO), in the monitoring of food spoilage and in the detection of frauds [65, 66]. Also, highly specific sensors and electronic tongues can be designed for the detection and quantification of relevant components of certain foods such as capsaicin in hot paprika, cinnamaldehyde in cinnamon or safranal in saffron. At this point, we should not forget that liquid chromatography with amperometric detection can take advantage of organic substances easily oxidizable that are characteristic of foods. The typical example of that are phenolic substances, which are present in many foods and beverages. Hence, amperometric detection can extract chromatographic fingerprints to be used in the discrimination of food samples [67].

We do not know which one of these fields (and other not discussed here) will be the most productive for electroanalysis. However, all of them face important challenges that we will try to summarize in the next section.

\section{The challenges of the new century}

The research lines above described require relevant advances in some specific areas to be really significant:

Supports for modified electrodes should be more versatile. For instance, most commercial screen-printed devices are too rigid for some applications such as wearable sensors [68], which can be important not only for medical applications but also for sport and military purposes. Some efforts have been made in this direction by using flexible materials and paper [69] or applying the methodology of hydrographic printing [70]. 3D printing is being investigated also as a new strategy to produce more sophisticated sensors [71]. This search for new supports is not only trying to improve versatility but also to reduce production costs and allow recycling of the used devices.

Non-invasive measurements with strategies like impedimetric sensing [72] should be developed and applied to many fields, with special attention to biomedical applications.

New materials, selective reagents, electrochemical mediators and modification strategies should be incorporated to the already extensive catalogue in order to improve both the selectivity and the sensitivity of existing electrochemical sensors [73]. This may come from both the research lines related to biomedicine (we suppose even more stimulated and funded since the appearance of covid-19) or from these related to 
nanoscience, which, encouraged by the extraordinary properties shown by carbon nanoallotropes, are investigating similar nanostructures in other elements such as silicon, phosphorous or bismuth.

Solid state electrochemistry is a discipline created to a great extent by Prof. Scholz some years ago $[11,74,75]$ that has proven to be especially useful for the analysis of solid samples (e.g. archaeological objects) as well as particles, droplets and vesicles placed on the electrode surface [76-79]. Having this in mind, solid state electrochemistry can experience a great expansion in the future to compete with other techniques like near-infrared spectroscopy and X-ray fluorescence for the direct analysis of solid samples. Obviously, the possibility of sensing directly on solid samples is a great added value to become a reliable complement to more sophisticated techniques that require the dissolution of the solid.

Electrochemical information should be enriched with other types of instrumental data in the so-called hybrid sensors. Spectroelectrochemistry can be quite valuable for that, since it provides simultaneous current and absorbance/reflectance measurements as potential and wavelengths change [80]. Moreover, some smart nanomaterials such as silver nanoparticles or graphene quantum dots present both electrochemical and spectroscopic properties that can be combined for this purpose [81-83]. Anyway, it is not strictly necessary that the different types of measurements are made simultaneously. The strategy of data fusion can be carried out in many ways.

Sensors should be miniaturized and integrated into operative sensor arrays. There, some of the sensors could individually detect relevant analytes, whereas other could take profit of their cross response towards some substances to act as electronic tongues in the detection of substances or the discrimination of samples [84]. Although much handmade research has been carried out in this direction so far, in the future, we should witness the mass production and commercialization of devices based on these principles.

Monitoring in flow systems has to be developed if electrochemical sensors aspire to be useful in on-site and/or on-line monitoring for environmental or industrial purposes. This can be made at regular scale or by using microfluidics [85-88]. In any case, the prevention against suspended particles and certain substances that could block the channels and cause electrode fouling is a matter of highest priority. Also, it is very important to ensure the durability of the sensing material, either by using highly resistant solids as BDD [25] or by ensuring a fast and automatic replacement of the sensor surface (e.g., by using a rotating roll of disposable SPE on paper). Finally, in the case of low concentration of the analytes, chemical and/or electrochemical preconcentration steps should be included in the methodology.

Wireless sensors have to play an essential role in the future, as they become integrated in different configurations of wireless sensor networks (WSN) powered by $5 \mathrm{G}$ technology and
Internet of things (IoT) [89]. The purposes of such networks can be very diverse. They can be used, for instance, to monitor patients at the points of care (POC) level, so analysis can be performed at any place outside the hospital by using portable kits or wearable sensors [90]. In a similar way, they can evaluate in real time the physical condition of athletes or sport players as they train or compete. In the environmental field, existing WSN designed to provide elementary information in the agricultural context (temperature, humidity...) should incorporate powerful chemical sensors able to report on real time the concentration of key compounds [91]. Obviously, important hardware and software developments would be required to acquire and process a large volume of data in the cloud and submit this to control rooms and/or portable devices such as laptops or smartphones.

In a future where sensor arrays and sensor networks will provide a large volume of data (frequently in real time), chemometric techniques that we use now could get insufficient and new methodologies, based on artificial intelligence and big data would have to be adapted or developed [92].

\section{Conclusions}

In this short and personal travel along the past, present and expected future of electroanalysis, we have the feeling that this discipline has been always making a compromise between the rationalistic trends of physical chemistry and the empirical trends of analytical chemistry. Electroanalysis has been able to survive many crises; thanks to a strong compromise to solve real problems and because of its dynamic interaction with many other disciplines (biochemistry, materials science, mathematics, computer science, chemometrics, electronics...). The key contributions of Prof. Scholz are a good example of this. Thus, we hope that this booming time of sensors could be really productive and keep electroanalysis in a prominent position inside analytical chemistry.

Funding information The authors acknowledge the support of the Generalitat of Catalonia (Project 2017SGR311), the Faculty of Chemistry of the University of Barcelona and the Water Research Institute (IdRA) of the University of Barcelona.

\section{References}

1. The Editors of Encyclopedia Britannica (2020) Philosopher's stone. https://www.britannica.com/topic/philosophers-stone

2. Bard AJ, Faulkner LR (2001) Electrochemical methods. Fundamentals and applications. 2nd Ed. Wiley, New York

3. Wang J (2006) Analytical electrochemistry. John Wiley, Hoboken

4. Bard AJ, Inzelt G, Scholz F Eds. (2008) Electrochemical dictionary. Series: Monographs in Electrochemistry, Springer, Berlin 
5. Scholz F Ed. (2010) Electroanalytical methods. Guide to experiments and applications. $2^{\text {nd }}$ Ed. Series: Monographs in Electrochemistry, Springer, Berlin

6. Breitkopf C, Swider-Lyons K (2017) Electrochemical sciencehistorical review. In: springer handbook of electrochemical energy. Springer, Berlin Heidelberg, pp 1-9

7. History of electrochemistry: Wikipedia Category, http:// commons.wikimedia.org/wiki/Category:History of_electrochemistry (2015)

8. Lubert KH, Kalcher K (2010) History of electroanalytical methods. Electroanalysis 22(17-18):1937-1946

9. Inzelt $\mathrm{G}$ (2020) Future of electrochemistry in light of history and the present conditions. J Solid State Electrochem 2020:1-4

10. Kolthoff IM (1954) Relations between voltammetry and potentiometric and amperometric titrations. Anal Chem 26(11):1685-1691

11. Scholz F, Meyer B (1998) Voltammetry of solid microparticles immobilized on electrode surfaces. In: Bard AJ, Rubinstein I (eds) Electroanalytical chemistry a series of advances: volume 20. Marcel Dekker, New York, pp 1-86

12. Heyrovský J (1922) "Elektrolysa se rtutovou kapkovou Kathodu"Chem Listy 16:256-264

13. Faridbod F, Reza Ganjali M, Dinarvand R, Norouzi P (2007) The fabrication of potentiometric membrane sensors and their applications. Afr J Biotechnol 6:2960-2987

14. Bratov A, Abramova N, Ipatov A (2010) Recent trends in potentiometric sensor arrays - a review. Anal Chim Acta 678(2):149-159

15. Mimendia A, Gutiérrez JM, Leija L, Hernández PR, Favari L, Muñoz R, del Valle M (2010) A review of the use of the potentiometric electronic tongue in the monitoring of environmental systems. Environ Model Softw 25(9):1023-1030

16. Barek J, Zima J (2003) Eighty years of polarography - history and future. Electroanalysis 15(5-6):467-472

17. Heyrovský J, Kůta J (2013) Principles of polarography. Elsevier, Amsterdam

18. Scholz F Ed. (2015) Electrochemistry in a divided world. Innovations in Eastern Europe in the $20^{\text {th }}$ century. Series: monographs in electrochemistry, Springer, Berlin

19. Molina A, González J (2016) Pulse voltammetry in physical electrochemistry and electroanalysis. Theory and applications. Series: monographs in electrochemistry, editor: Fritz Scholz, Springer, Berlin

20. Barek J, Fogg AG, Muck A, Zima J (2001) Polarography and voltammetry at mercury electrodes. Crit Rev Anal Chem 31(4):291309

21. Ariño C, Serrano N, Díaz-Cruz JM, Esteban M (2017) Voltammetric determination of metal ions beyond mercury electrodes. A review. Anal Chim Acta 990:11-53

22. Zoski CG (2002) Ultramicroelectrodes: design, fabrication, and characterization. Electroanalysis 14(15-16):1041-1051

23. Saravanakumar R, Pirabaharan P, Rajendran L (2019) The theory of steady state current for chronoamperometric and cyclic voltammetry on rotating disk electrodes for $\mathrm{EC}^{\prime}$ and $\mathrm{ECE}$ reactions. Electrochim Acta 313:441-456

24. Navratil T, Barek J (2009) Analytical applications of composite solid electrodes. Crit Rev Anal Chem 39(3):131-147

25. Chailapakul O, Siangproh W, Tryk DA (2006) Boron-doped diamond-based sensors: a review. Sens Lett 4(2):99-119

26. Wang J (2005) Stripping analysis at bismuth electrodes: a review. Electroanalysis 17(15-16):1341-1346

27. Economou A (2005) Bismuth-film electrodes: recent developments and potentialities for electroanalysis. Trac-Trend Anal Chem 24(4): 334-340

28. Serrano N, Díaz-Cruz JM, Ariño C, Esteban M (2016) Antimonybased electrodes for analytical determinations. Trac-Trend Anal Chem 77:203-213
29. Metters JP, Kadara RO, Banks CE (2011) New directions in screen printed electroanalytical sensors: an overview of recent developments. Analyst 136(6):1067-1076

30. Barton J, González García MB, Hernández Santos D, FanjulBolado P, Ribotti A, McCaul M, Diamond D, Magni P (2016) Screen-printed electrodes for environmental monitoring of heavy metal ions: a review. Microchim Acta 183(2):503-517

31. Domínguez Renedo O, Alonso-Lomillo MA, Arcos Martínez MJ (2007) Recent developments in the field of screen-printed electrodes and their related applications. Talanta 73(2):202-219

32. Li M, Li YT, Li DW, Long YT (2012) Recent developments and applications of screen-printed electrodes in environmental assaysa review. Anal Chim Acta 734:31-44

33. Taleat Z, Khoshroo A, Mazloum-Ardakani M (2014) Screenprinted electrodes for biosensing: a review (2008-2013). Microchim Acta 181(9-10):865-891

34. Honeychurch KC, Hart JP (2003) Screen-printed electrochemical sensors for monitoring metal pollutants. Trac-Trend Anal Chem 22(7):456-469

35. Serrano N, Alberich A, Díaz-Cruz JM, Ariño C, Esteban M (2013) Coating methods, modifiers and applications of bismuth screenprinted electrodes. Trac-Trend Anal Chem 46:15-29

36. Marcinkowska M, Barałkiewicz D (2016) Multielemental speciation analysis by advanced hyphenated technique-HPLC/ICP-MS: a review. Talanta 161:177-204

37. Korfmacher WA (2005) Foundation review: principles and applications of LC-MS in new drug discovery. Drug Discov Today 10(20):1357-1367

38. Zhou S, Song Q, Tang Y, Naidong W (2005) Critical review of development, validation, and transfer for high throughput bioanalytical LC-MS/MS methods. Curr Pharm Anal 1(1):3-14

39. Brett CM, Oliveira-Brett AM (2011) Electrochemical sensing in solution-origins, applications and future perspectives. J Solid State Electrochem 15(7-8):1487-1494

40. Seeber R, Terzi F (2011) The evolution of amperometric sensing from the bare to the modified electrode systems. J Solid State Electrochem 15(7-8):1523-1534

41. Yoo EH, Lee SY (2010) Glucose biosensors: an overview of use in clinical practice. Sensors 10(5):4558-4576

42. Zhang X, Ju H, Wang J (eds) (2008) Electrochemical sensors, biosensors and their biomedical applications, Elsevier. San Diego, CA, USA

43. DeFord DD, Hume DN (1951) The determination of consecutive formation constants of complex ions from polarographic data. J Am Chem Soc 73(11):5321-5322

44. Díaz-Cruz JM, Esteban M, Ariño C (2019) Chemometrics in electroanalysis. Inside 'Monographs in Electrochemistry', edited by F. Scholz, Springer Nature, Cham, Switzerland

45. Esteban M, Ariño C, Díaz-Cruz JM (2006) Chemometrics for the analysis of voltammetric data. Trac-Trend Anal Chem 25(1):86-92

46. Richards E, Bessant C, Saini S (2002) Multivariate data analysis in electroanalytical chemistry. Electroanalysis 14(22):1533-1542

47. Esteban M, Ariño C, Díaz-Cruz JM, Díaz-Cruz MS, Tauler R (2000) Multivariate curve resolution with alternating least squares optimisation: a soft-modelling approach to metal complexation studies by voltammetric techniques. Trac-Trend Anal Chem 19(1):49-61

48. Ciosek P, Wróblewski W (2011) Potentiometric electronic tongues for foodstuff and biosample recognition - an overview. Sensors 11(5):4688-4701

49. Díaz-Cruz JM, Pérez-Ràfols C, Cetó X, Serrano N, Ariño C, Esteban M (2019) Voltammetric electronic tongues in recent advances in analytical techniques, vol 4, 68-98. Bentham Science, United Arab Emirates

50. http://www.ciencia.gob.es/stfls/MICINN/Ministerio/FICHEROS/ TecnicasDiagnosticoCOVID19-ICN2.pdf (accessed April 9, 2020) 
51. Van Dorst B, Mehta J, Bekaert K, Rouah-Martin E, De Coen W, Dubruel P, Blust R, Robbens J (2010) Recent advances in recognition elements of food and environmental biosensors: a review. Biosens Bioelectron 26(4):1178-1194

52. Fletcher S (2011) Electronomics. J Solid State Electrochem 15(78):1451-1458

53. Guidelli R, Becucci L (2011) Ion transport across biomembranes and model membranes. J Solid State Electrochem 15(7-8):1459 1470

54. Chen A, Chatterjee S (2013) Nanomaterials based electrochemical sensors for biomedical applications. Chem Soc Rev 42(12):54255438

55. Arduini F, Micheli L, Moscone D, Palleschi G, Piermarini S, Ricci F, Volpe G (2016) Electrochemical biosensors based on nanomodified screen-printed electrodes: recent applications in clinical analysis. Trac-Trend Anal Chem 79:114-126

56. Reverté L, Prieto-Simón B, Campàs M (2016) New advances in electrochemical biosensors for the detection of toxins: nanomaterials, magnetic beads and microfluidics systems. A review. Anal Chim Acta 908:8-21

57. Ambhorkar P, Wang Z, Ko H, Lee S, Koo K, Kim K, Cho D (2018) Nanowire-based biosensors: from growth to applications. Micromachines 9, 679:19 pages

58. Pichon V, Chapuis-Hugon F (2008) Role of molecularly imprinted polymers for selective determination of environmental pollutantsa review. Anal Chim Acta 622(1-2):48-61

59. Sunyer A, González-Navarro A, Serra-Roig MP, Serrano N, DíazCruz MS, Díaz-Cruz JM (2019) First application of carbon-based screen-printed electrodes for the voltammetric determination of the organic UV filters oxybenzone and octocrylene. Talanta 196:381388

60. Serrano N, Castilla O, Ariño C, Díaz-Cruz MS, Díaz-Cruz JM (2019). Commercial screen-printed electrodes based on carbon nanomaterials for a fast and cost-effective voltammetric determination of paracetamol, ibuprofen and caffeine in water samples. Sensors 19, 4039:16 pages

61. Pérez-Ràfols C, Serrano N, Díaz-Cruz JM, Ariño C, Esteban M (2016) New approaches to antimony film screen-printed electrodes using carbon-based nanomaterials substrates. Anal Chim Acta 916: $17-23$

62. Inzelt G (2017) Recent advances in the field of conducting polymers. J Solid State Electrochem 21(7):1965-1975

63. Putra BR, Harito C, Bavykin DV, Walsh FC, Wahyuni WT, Boswell JA et al (2019) Processes associated with ionic current rectification at a 2D-titanate nanosheet deposit on a microhole poly (ethylene terephthalate) substrate. J Solid State Electrochem 23(4): $1237-1248$

64. Nasir MZM, Pumera M (2019) Emerging mono-elemental 2D nanomaterials for electrochemical sensing applications: from borophene to bismuthene. Trac-Trend Anal Chem 121, 115696:9 pages

65. Ghasemi-Varnamkhasti M, Apetrei C, Lozano J, Anyogu A (2018) Potential use of electronic noses, electronic tongues and biosensors as multisensor systems for spoilage examination in foods. Trends Food Sci Technol 80:71-92

66. Wadehra A, Patil PS (2016) Application of electronic tongues in food processing. Anal Methods 8(3):474-480

67. Serrano N, Cetó X, Núñez O, Aragó M, Gámez A, Ariño C, DíazCruz JM (2018) Characterization and classification of Spanish paprika (Capsicum annuum L.) by liquid chromatography coupled to electrochemical detection with screen-printed carbon-based nanomaterials electrodes. Talanta 189:296-301
68. Bandodkar AJ, Wang J (2014) Non-invasive wearable electrochemical sensors: a review. Trends Biotechnol 32(7):363-371

69. Silveira CM, Monteiro T, Almeida MG (2016) Biosensing with paper-based miniaturized printed electrodes-a modern trend. Biosensors 6:51:17

70. Zeng X, Dong Y, Wang X (2020) Flexible electrode by hydrographic printing for surface electromyography monitoring. Materials 13(10):2339

71. Muñoz J, Pumera M (2020) 3D-printed biosensors for electrochemical and optical applications. Trac - Trends in Analytical Chemistry 115933

72. Magar HS, Abbas MN, Ali MB, Ahmed MA (2020) Picomolarsensitive impedimetric sensor for salivary calcium analysis at POC based on SAM of Schiff base-modified gold electrode. J Solid State Electrochem 24(3):723-737

73. Pérez-Ràfols C, Rosal M, Serrano N, Ariño C, Esteban M, DíazCruz JM (2019) Expanding the possibilities of electrografting modification of voltammetric sensors through two complementary strategies. Electrochim Acta 319:878-884

74. Scholz F, Meyer B (1994) Electrochemical solid state analysis: state of the art. Chem Soc Rev 23(5):341-347

75. Grygar T, Marken F, Schröder U, Scholz F (2002) Electrochemical analysis of solids. A review. Coll Czech Chem Commun 67(2): 163-208

76. Scholz F (2011) The electrochemistry of particles, droplets, and vesicles-the present situation and future tasks. J Solid State Electrochem 15(7-8):1699-1702

77. Domenech-Carbó A (2017) Electrochemical dating: a review. J Solid State Electrochem 21(7):1987-1998

78. Doménech-Carbó A, Scholz F, Doménech-Carbó MT, PiqueroCilla J, Montoya N, Pasíes-Oviedo T, Oliver A (2018) Dating of archaeological gold by means of solid state electrochemistry. ChemElectroChem 5(15):2113-2117

79. Hümmelgen IA (2017) Organic electronic solid state device: electrochemistry of material preparation. J Solid State Electrochem 21(7):1977-1985

80. González-Diéguez N, Colina A, López-Palacios J, Heras A (2012) Spectroelectrochemistry at screen-printed electrodes: determination of dopamine. Anal Chem 84(21):9146-9153

81. Luo J, Yao J, Lu Y, Ma W, Zhuang X (2013) A silver nanoparticlemodified evanescent field optical fiber sensor for methylene blue detection. Sensors 13(3):3986-3997

82. Faridbod F, Sanati AL (2019) Graphene quantum dots in electrochemical sensors/biosensors. Curr Anal Chem 15(2):103-123

83. Ting SL, Ee SJ, Ananthanarayanan A, Leong KC, Chen P (2015) Graphene quantum dots functionalized gold nanoparticles for sensitive electrochemical detection of heavy metal ions. Electrochim Acta 172:7-11

84. Del Valle M (2010) Electronic tongues employing electrochemical sensors. Electroanalysis 22:1539-1555

85. Aogaki R, Ito E, Ogata M (2007) A new flow-type cell by the application of magnetic microfluidic chip. J Solid State Electrochem 11(6):757-762

86. Jia Y, Bi A, Selimovic A, Martin RS, Kiss IZ (2015) Periodic and complex waveform current oscillations of copper electrodissolution in phosphoric acid in an epoxy-based microchip flow cell. J Solid State Electrochem 19(11):3241-3251

87. Bîrzu A, Kiss IZ (2015) Asymmetrical multiphase front propagation and localized oscillations in a reaction-migration iron electrodissolution model with microfluidic flow cell geometry. J Solid State Electrochem 19(11):3229-3240 
88. Salim A, Lim S (2018). Review of recent metamaterial microfluidic sensors. Sensors 18, 232:25 pages

89. Ruiz-Garcia L, Lunadei L, Barreiro P, Robla I (2009) A review of wireless sensor technologies and applications in agriculture and food industry: state of the art and current trends. Sensors 9(6): $4728-4750$

90. Malasinghe LP, Ramzan N, Dahal K (2019) Remote patient monitoring: a comprehensive study. J Ambient Intell Humaniz Comput 10(1):57-76
91. Ojha T, Misra S, Raghuwanshi NS (2015) Wireless sensor networks for agriculture: the state-of-the-art in practice and future challenges. Comput Electron Agric 118:66-84

92. Al-Jarrah OY, Yoo PD, Muhaidat S, Karagiannidis GK, Taha K (2015) Efficient machine learning for big data: a review. Big Data Res 2(3):87-93

Publisher's note Springer Nature remains neutral with regard to jurisdictional claims in published maps and institutional affiliations. 